\title{
The Quality Evaluation Method Research on Cloud Cover Image Based on Statistical Analysis
}

\author{
Yanjie Wang, Jianjun Zhou, Li Jia \\ The Unit of 91635th, PLA, Beijing, China \\ email:2742069961@qq.com
}

\begin{abstract}
Keywords: Cloud cover; Image quality evaluation; Statistical model; Geometry dimensions; Angular second moment
\end{abstract}

\begin{abstract}
Cloud cover is a key factor which affects the quality of aerial and remote sensing image. In order to precisely evaluate the impact which brought by cloud cover, article constructs a cloud detection algorithm which suits for aerial image according to cloud statistical characters; analyzing the cloud region, and building a model on the basis of the image quality comprehensive assessment of the thick cloud, thin cloud, possible cloud and cloudless region; at last, utilizing experts' subjective guidance to improve the evaluation model. The algorithm can efficiently and precisely evaluate the aerial cloud cover image on the account massive test data.
\end{abstract}

\section{Introduction}

Image is an important way for human to acquire information. Therefore, constructing image quality evaluation mechanism is significant[1][2]. Along with development of aviation technique and simulation, aerial image application is used more and more widely. However, due to many impact factors such as height and climate, it's hard to get the image without any cloud cover. Cloud cover has become main impact factor of affecting aerial image quality. Evaluating cloud cover image is valuable for aviation image application and process tools.

So far, the normal method of evaluating cloud cover image is that firstly detect cloud cover region and then evaluate the quality of this region[3]. Cloud cover region detection method can be divided into two types: first type is spectrum analysis method which using reflectance value which get by multi-channel data acting on cloud and land, and second is using cloud's texture statistical feature; article takes no reference methods such as PSNR, SSIM, VIF[4][5] as criteria calculation. Because of the roughness of the detection result, simplicity of the model, article got a low accuracy of evaluation result and not well performed.

For the above application requirements, article combines statistic methods with meticulous classification for the cloud region and using learning theories to improve the evaluation model and construct more accurate objective evaluation model.

\section{Feature analysis}

In the field of cloud detection, useful detection feature include: grayscale, shape, texture, shadow area six types features. Different types of clouds have different feature value because of height, temperature, climate etc. Therefore, we use mathematic statistic methods to do multi-feature evaluation at cloud region in order to get more accurate detection result.

In the field of image evaluation, there are two general types: subjective method and objective method. The former method relies on humans' subjective perception to evaluate image; the latter method evaluate image according to quantitative criteria, for instance: PSNR, SSIM, VIF etc. Subjective quality evaluation is the most reliable method but restricted by human power, time cost, poor generalization[7]. Along with the development of AI, we can use the result of subjective evaluation to guide the objective model to get a better evaluation method.

Article utilizes angle second order matrix and fractal dimension to detect and classify the cloud region as well as using experts' evaluation result and neural network to build evaluation model, deal 
process as below:

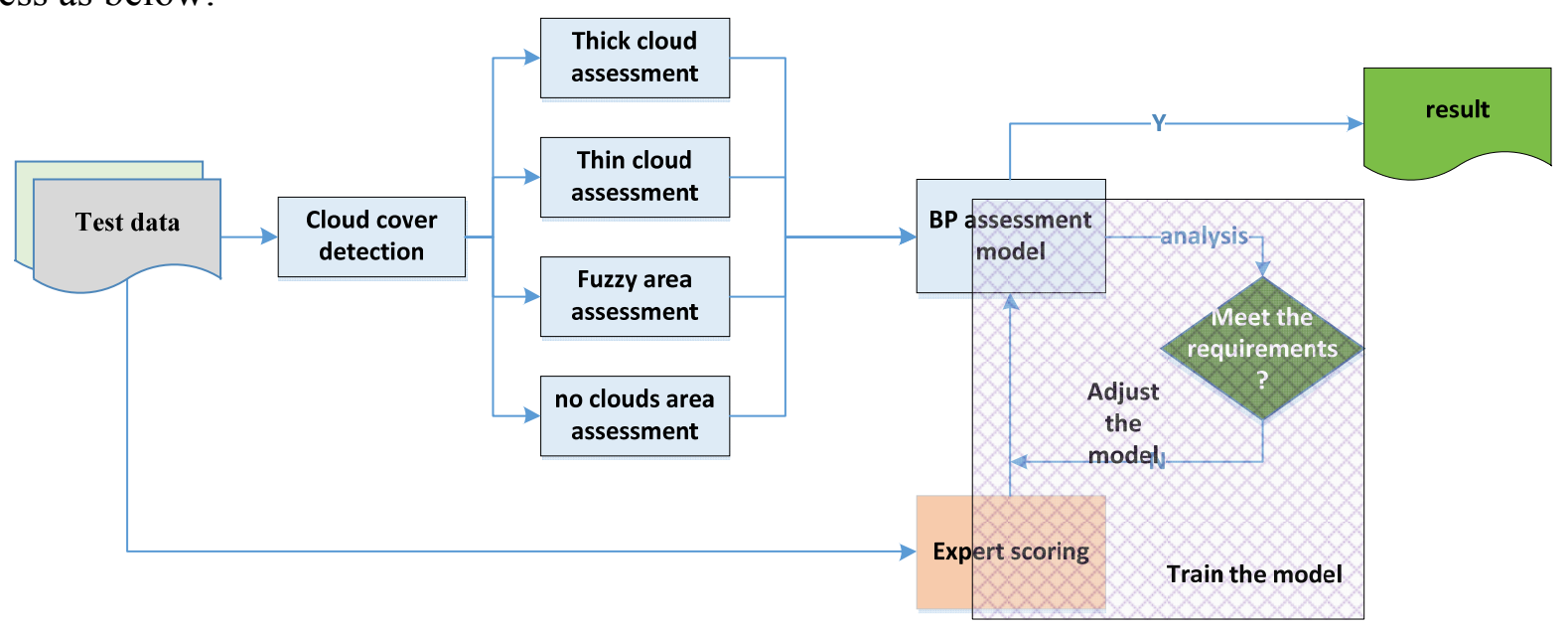

Fig.1. Clouds cover image quality assessment procedure

\section{Algorithm procedure}

\section{Clouds area detection}

Firstly, randomly select the images which contain clouds and extract the little areas with or without clouds to constitute a sample data set. Secondly, analysis the data set to get the four kinds of images' gray distribution range and variance. Statistical analysis of fractal dimension data set, we get four areas of general interval; Thirdly, statistical analysis the datasets of angular second moment, get four areas roughly range. Cross use the intervals and find out the class the cloud areas.

The clouds area and other areas' difference in the image gray level is one of the most obvious distinguish characteristic, therefore, we use gray characteristics for a preliminary inspection of cloud area.

\section{Clouds area segmentation}

Fractal dimension reflects the self-similarity of fractal surface, is used to distinguish between different types of things. According to the interval of the fractal dimension value, determine the thick clouds, and find out the images without clouds in the thin clouds images data sets.

Fractal dimension range determination method:

1) Transform a $\mathrm{M} * \mathrm{M}$ two-dimensional gray image into a three-dimensional image, which $\mathrm{f}(\mathrm{x}$, $\mathrm{y})$ is the grey value of $(\mathrm{x}, \mathrm{y})$.

2) Split the images with $r^{*} r$ grid, in a three-dimensional image $\left(x, y, f(x, y)\right.$ will generate $r^{*} r^{*} h$ small cubes, $h=r^{*} \mathrm{G} / \mathrm{M}$, which $\mathrm{G}$ is the number of different gray value in the cube.

3) Assumption that the minimum gray value is in the cube $\mathrm{k}$, and the maximum gray value is in the cube $\mathrm{m}$. Thus the minimum number of the cube that can contain all the gray value of grid is:

$$
\mathrm{n}(\mathrm{i}, \mathrm{j})=\mathrm{m}-\mathrm{k}+1
$$

4) Recalculate the cube number with this formula:

$$
N r=\sum_{i, j} n(i, j)
$$

5) Calculate the fractal dimension with this formula:

$$
D=\lim (\log (N r) / \log (1 / r))
$$

Which means using the $\log (1 / \mathrm{r})$ as the abscissa, $\log (\mathrm{Nr})$ as the ordinate, and using the least squares fitting of this lattice slope of the graph.

Through analysis, we can come to the conclusion that: thick clouds' range is $1.89 \sim 2.45$, thin clouds' is $1.4 \sim 2.45$, no clouds images' $2.45 \sim 2.75$. Aviation image cloud-free area corresponding to the underlying surface of the object type is relatively complex, grey value distribution range is large, 
trait of texture details is obvious, the fractal dimension value is larger; On the contrary, the cloud area has relatively small changes, smooth surface, the smaller the fractal dimension value.

\section{Clouds area determination}

Angle values of second moment can measure the uniformity of gray level distribution, so as to the identification of a wide range of similar goals. According to the distribution range of values of angular second moment, we can find out the thick clouds which is judged to be thin clouds and the thin clouds which is judged to be no clouds. The formula of calculating angle values of second moment is:

$$
A S M=\sum_{h=0}^{M-1} \sum_{k=0}^{N-1}\left(m_{h k}\right)^{2}
$$

where $m_{h k}$ is the value of the normalization of gray level co-occurrence matrix in position (h,k).

By analysis the sample data, we get the distribution space of angular second moment values: the thick clouds' image $0.95 \sim 1.05$, the thin clouds' is $0.22 \sim 1.05$, no clouds' is $0.02 \sim 1.05$.Furthure analysis, Thick cloud contains more energy, the angular second moment value is larger and almost concentrated near to 1; Thin clouds' energy is smaller than thick clouds, so the value is focus on higher value of 1 , Part of the angular second moment value distribution between $0.22 \sim 0.83$; No clouds areas' texture fine, Angular second moment value is uniformly distributed in the range of $0.02 \sim 0.70$.

The classification procedure of detection algorithm is as follows:

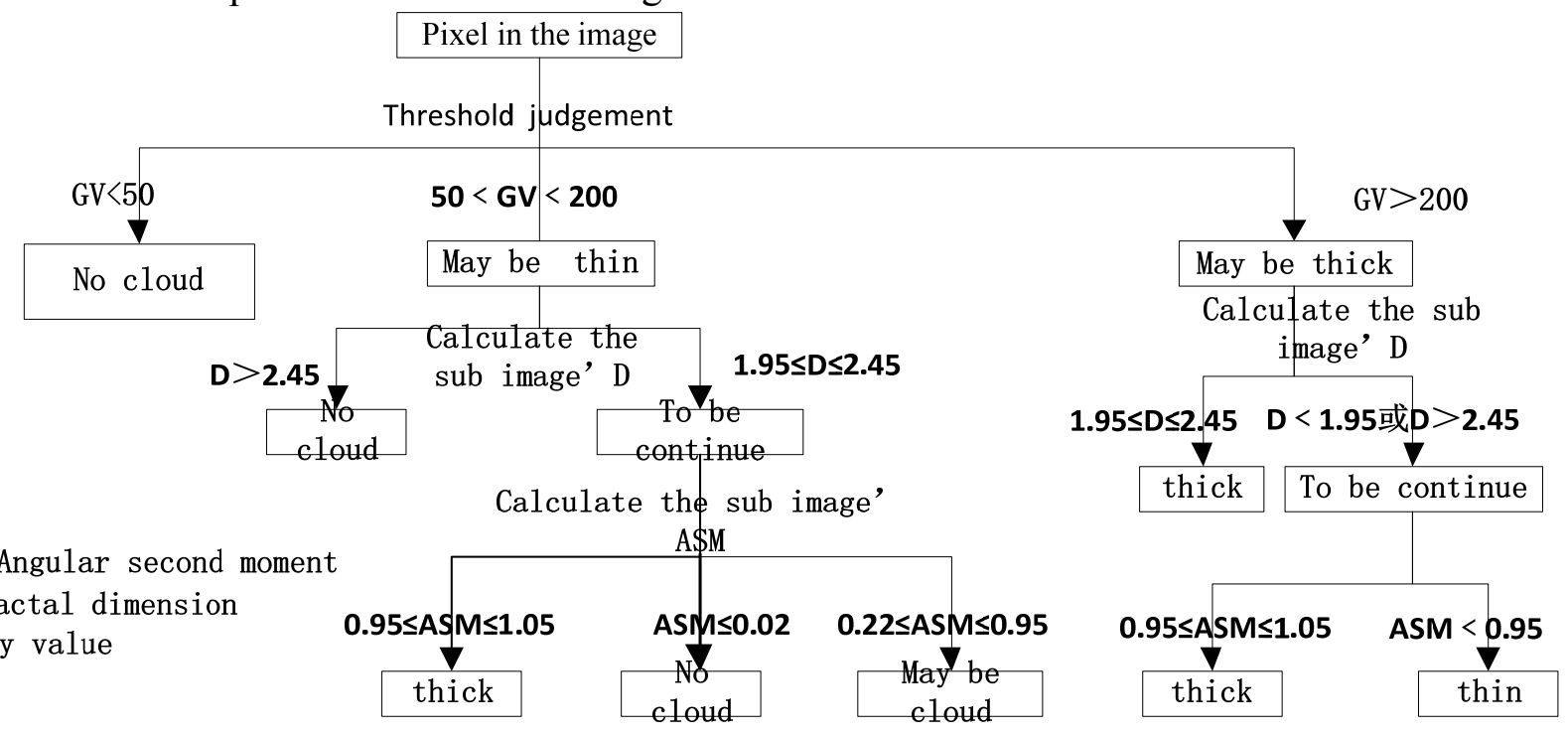

Fig.2. The classification procedure of detection algorithm

\section{Clouds area image quality assessment}

After getting the result, we can calculate different cloud area ratio to evaluate image quality[8]. The area of the thick cloud detected in the image is $S_{\text {thick }}$, the area of thin cloud is $S_{\text {thin }}$, the area of aerial reconnaissance image is $\mathrm{m}^{*} \mathrm{n}$, the cloud area of the image area ratio in the image is:

$$
\left\{\begin{array}{l}
\gamma_{\text {thick }}=S_{\text {thick }} /(m \times n) \\
\gamma_{\text {thin }}=S_{\text {thin }} /(m \times n) \\
\gamma_{\text {possible }}=S_{\text {possible }} /(m \times n)
\end{array}\right.
$$

Firstly, build a three layers BP network, input features are the three kinds of covered areas and uncovered areas, and output feature is a Comprehensive quality assessment result. Thus, BP network's input layer is four neurons, hidden layer is eight neurons, output layer is a neurons.

The training data set is made up of 200 simulation images and 400 real images. The 200 
simulation images are viewed as a standard test image, providing its subjective grading standard, which are used as a validation of BP network. The 400 real images' score is provided by 15 observers. According to the subjective evaluation method given by ITU - R BT. 500-13 proposal[9], we use a single incentive method to calculate a subjective score. We train the assessment model with the data, and make use of the model to assess the cloud cover images without reference.

\section{Experimental results and analysis}

To test the image quality objective evaluation results and the consistency of subjective perception, using Pearson Correlation Coefficient PCC (Pearson Linear Correlation Coefficient) to evaluate:

$$
\mathrm{PCC}=\frac{\sum_{i=1}^{n}\left(X_{i}-\bar{X}\right)\left(Y_{i}-\bar{Y}\right)}{\sqrt{\sum_{i=1}^{n}\left(X_{i}-\bar{X}\right)^{2} \sum_{i=1}^{n}\left(Y_{i}-\bar{Y}\right)^{2}}}
$$

Where $\mathrm{X}$ and $\mathrm{Y}$, respectively subjective assessment and objective assessment results, $\mathrm{N}$ as the sample.

By collecting public image and organizing internal data, we have more than 1000 distortion image for validation, including thick clouds, thin clouds, there may be a cloud and there may not be a cloud.

Each of the distortion type has 200 standard images (with subjective quality score) and have different intensity of distortion image, part of the content of the test data as shown below:

Clouds cover distortion images without reference:

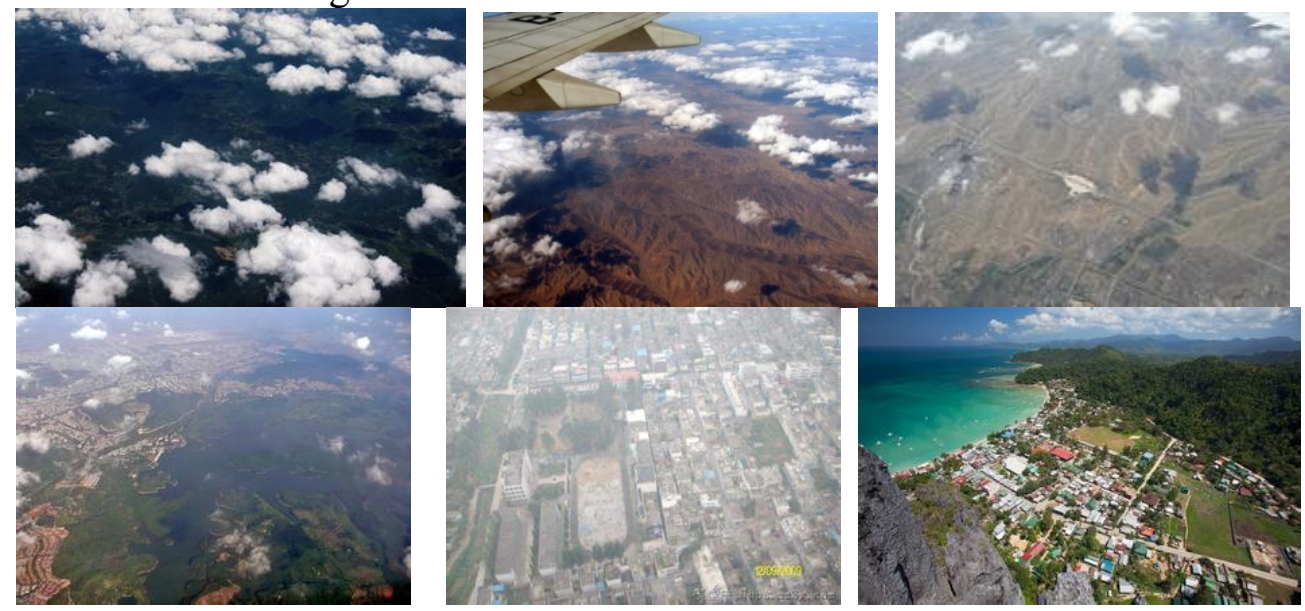

Fig.3.clouds cover images in different level

We randomly select 200 clouds cover distortion aviation image (with the subjective score), by using a quality evaluation model, we calculate the objective score without reference images.

The final results of images are shown in Table 1.The Subjective and objective assessment result of 200 images is shown in Figure 4. Pearson correlation coefficient is 0.82 and average Computation time is 1.5 seconds per image.

Table. 1 The assessment results of clouds cover distortion images

\begin{tabular}{|c|c|c|c|c|c|c|c|c|c|c|c|}
\hline S & Su & Ob & S & Su & Ob & S & Su & Ob & S & Su & Ob \\
\hline 01 & 1.6 & 1.3 & 06 & 4.0 & 3.4 & 11 & 6.0 & 5.1 & 16 & 7.4 & 7.5 \\
\hline 02 & 2.0 & 1.5 & 07 & 4.4 & 3.9 & 12 & 6.4 & 5.3 & 17 & 7.6 & 7.9 \\
\hline 03 & 2.8 & 2.3 & 08 & 4.8 & 4.2 & 13 & 6.8 & 6.5 & 18 & 7.8 & 8.1 \\
\hline 04 & 3.2 & 2.7 & 09 & 5.0 & 4.5 & 14 & 7.0 & 7.2 & 19 & 8.0 & 8.1 \\
\hline 05 & 3.6 & 3.0 & 10 & 5.4 & 5.5 & 15 & 7.2 & 7.4 & 20 & 8.2 & 8.4 \\
\hline
\end{tabular}

( S:sequence,Su:subjective,Ob:objective ) 


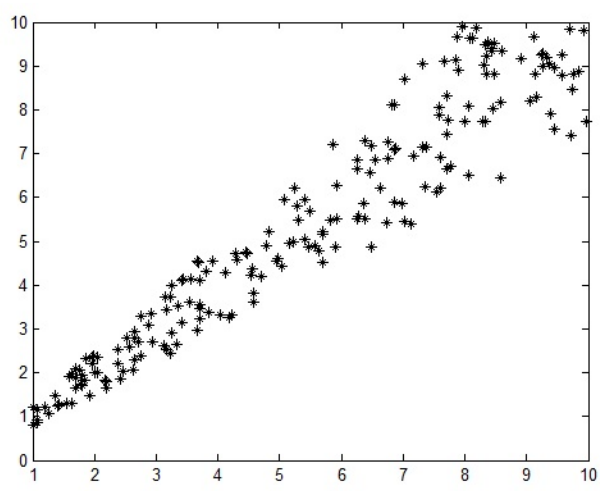

Fig.4. The subjective and objective assessment result of 200 images

\section{Conclusion}

This paper puts forward an effective cloud cover image quality assessment method, firstly, divide the image into different areas by a variety of statistical features, train the BP assessment model by using expert scoring criteria result image, then using the model of image quality evaluation to get the final score. The results on the actual database show that the algorithm in this paper and expert scoring high fit, convenient calculation.

Image quality assessment is an important problem in the field of image processing technology, which requires the assessment result is consistent of the visual perceptual of people and easy to calculate. Considering the importance of image quality assessment, the subsequent work will focus on the more general fuzzy, noise, degradation studies as well as the quality problems, building general evaluation model, so as to adapt to the application of the more common problems.

\section{References}

[1] M. A. Saad, A. C. Bovik, and C. Charrier, "Blind image quality assessment: a natural scene statistics approach in the DCT domain," IEEE Trans. Image Process., vol. 21, no. 8, pp. 3339-3352, Aug. 2012.

[2] A. K. Moorthy and A. C. Bovik, "Blind image quality assessment: From natural scene statistics to perceptual quality," IEEE Trans. Image Process., vol. 20, no. 12, pp. 3350-3364, Dec. 2011.

[3] A. K. Moorthy and A. C. Bovik, "A two-step framework for constructing blind image quality indices,” IEEE Signal Process. Lett., vol. 17, no. 5, pp. 513-516, May. 2010.

[4] X. Gao, F. Gao, D. Tao, and X. Li, "Universal Blind Image Quality Assessment Metrics Via Natural Scene Statistics and Multiple Kernel Learning," IEEE Trans. Neural Networks and Learning Systems, vol. 24, no. 12, pp. 2013-2026, Dec. 2013.

[5] A. Mittal, A. K. Moorthy and A. C. Bovik, "No-reference image quality assessment in the spatial domain,” IEEE Trans. Image Process., vol. 21, no. 12, pp. 4695-4708, Dec. 2012.

[6] A. Mittal, R. Soundararajan and A. C. Bovik, "Making a 'Completely Blind' Image Quality Analyzer,” IEEE Signal Processing Letters, vol. 20, no. 3, pp. 209-212, Mar. 2013.

[7] J. Li, X. Zhang and M. Ding, "Image quality assessment based on regional mutual information", 2011 International Conference on Intelligent Computation and Bio-Medical Instrumentation.

[8] L. He, D. Tao, X. Li, and X. Gao, "Sparse representation for blind image quality assessment," IEEE Conference on Computer Vision and Pattern Recognition, pp. 1146-1153, Jun. 2012.

[9] H. R. Sheikh and A. C. Bovik, "Image information and visual quality," IEEE Trans. Image Process., vol. 15, no. 2, pp. 430-444, Feb. 2006. 